Integration of silicon-based neural probes and micro-drive arrays for chronic recording of large populations of neurons in behaving animals

This content has been downloaded from IOPscience. Please scroll down to see the full text.

2016 J. Neural Eng. 13046018

(http://iopscience.iop.org/1741-2552/13/4/046018)

View the table of contents for this issue, or go to the journal homepage for more

Download details:

IP Address: 94.224.118.140

This content was downloaded on 22/01/2017 at 16:01

Please note that terms and conditions apply.

You may also be interested in:

A silicon-based microelectrode array with a microdrive for monitoring brainstem regions of freely moving rats

G Márton, P Baracskay, B Cseri et al.

Fabrication technology for silicon-based microprobe arrays used in acute and sub-chronic neural recording

S Herwik, S Kisban, A A A Aarts et al.

Chronic neural probe for simultaneous recording of single-unit, multi-unit, and local field potential activity from multiple brain sites

F Pothof, L Bonini, M Lanzilotto et al.

An implantable neural probe with monolithically integrated dielectric waveguide and recording

electrodes for optogenetics applications

Fan Wu, Eran Stark, Maesoon Im et al.

3D Parylene sheath neural probe for chronic recordings

B J Kim, J T W Kuo, S A Hara et al.

3D probe array integrated with a front-end 100-channel neural recording ASIC

Ming-Yuan Cheng, Lei Yao, Kwan Ling Tan et al.

In-plane silicon probes for simultaneous neural recording and drug delivery

K Seidl, S Spieth, S Herwik et al.

Design, simulation and experimental validation of a novel flexible neural probe for deep brain stimulation and multichannel recording

Hsin-Yi Lai, Lun-De Liao, Chin-Teng Lin et al. 


\title{
Integration of silicon-based neural probes and micro-drive arrays for chronic recording of large populations of neurons in behaving animals
}

\author{
Frédéric Michon ${ }^{1,2}$, Arno Aarts ${ }^{3}$, Tobias Holzhammer ${ }^{3,4}$, Patrick Ruther ${ }^{4}$, \\ Gustaaf Borghs $^{1}$, Bruce McNaughton ${ }^{5}$ and Fabian Kloosterman ${ }^{1,6,7,8}$ \\ ${ }^{1}$ Neuro-Electronic Research Flanders (NERF), Leuven, Belgium \\ ${ }^{2}$ Department of Neuroscience, Biomedical Sciences, KULeuven, Belgium \\ ${ }^{3}$ ATLAS Neuroengineering, Leuven, Belgium \\ ${ }^{4}$ Department of Microsystems Engineering (IMTEK), University of Freiburg, Germany \\ ${ }^{5}$ Department of Neurosciences, Canadian Centre for Behavioral Neuroscience (CCBN), University of \\ Lethbridge, Canada \\ ${ }^{6}$ Brain \& Cognition Research Unit, KULeuven, Belgium \\ ${ }^{7}$ Imec, Leuven, Belgium \\ E-mail: fabian.kloosterman@nerf.be
}

Received 2 February 2016, revised 22 May 2016

Accepted for publication 7 June 2016

Published 28 June 2016

\begin{abstract}
Objective. Understanding how neuronal assemblies underlie cognitive function is a fundamental question in system neuroscience. It poses the technical challenge to monitor the activity of populations of neurons, potentially widely separated, in relation to behaviour. In this paper, we present a new system which aims at simultaneously recording from a large population of neurons from multiple separated brain regions in freely behaving animals. Approach. The concept of the new device is to combine the benefits of two existing electrophysiological techniques, i.e. the flexibility and modularity of micro-drive arrays and the high sampling ability of electrode-dense silicon probes. Main results. Newly engineered long bendable silicon probes were integrated into a micro-drive array. The resulting device can carry up to 16 independently movable silicon probes, each carrying 16 recording sites. Populations of neurons were recorded simultaneously in multiple cortical and/or hippocampal sites in two freely behaving implanted rats. Significance. Current approaches to monitor neuronal activity either allow to flexibly record from multiple widely separated brain regions (micro-drive arrays) but with a limited sampling density or to provide denser sampling at the expense of a flexible placement in multiple brain regions (neural probes). By combining these two approaches and their benefits, we present an alternative solution for flexible and simultaneous recordings from widely distributed populations of neurons in freely behaving rats.
\end{abstract}

Keywords: neural probe, neurophysiology, behaving rodents

(Some figures may appear in colour only in the online journal)

\section{Introduction}

Neuronal assemblies, distributed across multiple brain structures, are the main functional units for information processing

8 Author to whom any correspondence should be addressed. in the mammalian brain [1]. Hebb's hypothesis implies that looking at the behaviour of single neurons or the activity from single brain areas independently is not sufficient to understand brain functions. Understanding how neuronal activity supports cognition and complex behaviour requires the study of dynamically correlated activity in the populations of 
neurons that form assemblies. This poses an associated technological challenge: the simultaneous, large-scale readout of brain activity during behaviour in a representative sample of neurons in connected but potentially widely separated brain structures.

The extracellular measurement of electrical potentials that reflect transmembrane currents of nearby neural sources is a powerful and widely used approach to monitor the activity of neurons in vivo. For this purpose, single electrodes can be implanted in deep as well as superficial brain structures. They are capable of capturing both the combined activity of local populations of neurons (local field potential) and single cell activity (action potentials or spikes) at high temporal resolution.

The spikes of multiple neurons can be recorded on a single implanted electrode and the contributed spikes of each individual neuron can be classified on the basis of waveform shape and amplitude. However, only a limited number of units can be isolated from noise and other units in this way [2]. The yield (in terms of number of neurons) of the recording device and classification process can be enhanced by using multiple closely-spaced electrodes that sense a largely overlapping volume of tissue. Multi-wire probes, such as stereotrodes and tetrodes, increase the number of cells that can be reliably identified by effectively triangulating the signals across multiple electrodes [3].

More recently, advanced silicon processing technologies have been applied to the construction of needle-like probes carrying a dense array of electrodes along the probe shank [4-7]. The increased number of electrodes allows sampling from a larger number of neurons in the tissue volume around the probe than equivalently sized wire electrodes. Moreover and although it remains an open question, recording from electrode-dense probes with a high number of closely spaced electrodes may also enhance the triangulation of the signals [8] and the efficiency of the spike classification process.

Multiple electrodes or probes can be implanted at once to monitor the activity in more than one location simultaneously, either within a single brain structure or across several spatially separated areas. Arrays of screw-driven micro-drives provide a convenient way to position bundles of wire electrodes in the desired target region(s) $[9,10]$. Custom designed and manufactured micro-drive arrays provide two distinct advantages: (1) individual control over the depth of each electrode for accurate positioning in the targeted brain volume and (2) flexible and modular arrangement of the electrode bundles to hit distributed brain regions [11].

Without further miniaturisation, current micro-drive array designs scale up to approximately 16 or 40 individual tetrodes in mice and rats, respectively (for a total of 64-160 electrodes), before reaching the weight limit that the animals are capable of carrying on their head. This limitation forces to trade-off between the number of regions and the sampling of cells within each targeted region. Multi-shank silicon-based probes arranged into a two-dimensional or three-dimensional
(3D) grid [12-14] increase the total number of electrodes and provide dense sampling of a single brain region. Such arrays miss however the flexible positioning provided by microdrive arrays. Rather, our objective is to combine the advantages of both approaches and develop a new device that integrates long, bendable silicon probes into custom microdrive arrays to enable recording from large neuronal populations in multiple distributed brain areas in freely moving rodents.

\section{Methodology}

\section{Long, bendable silicon-based multi-electrode neural probes}

Probe design. For integration into a micro-drive array, probes were designed with a $40 \mathrm{~mm}$ long, $150 \mu \mathrm{m}$ wide and $50 \mu \mathrm{m}$ thick slender probe shaft attached to a $2.5 \times 0.64 \mathrm{~mm}$ base (figure 1(A)). The mechanical flexibility of the probe shaft is enhanced by the specific fabrication process described below. It allows the funnelling of multiple probes into a micro-drive array to create a high-density probe array.

The probe shafts carry 16 electrodes arranged in application specific layouts (figure 1(B)): either two columns of 8 electrodes (' $D$ '-style probe) or a combination of a cluster of electrodes and a linear array (' $L$ '-style probe). The electrodes have a surface area of either $100 \mu \mathrm{m}^{2}$ $\left(10 \times 10 \mu \mathrm{m}^{2}\right)$ or $225 \mu \mathrm{m}^{2}\left(15 \times 15 \mu \mathrm{m}^{2}\right)$ and are made from gold. The electrodes are electrically interfaced to bonding pads on the probe base via $3 \mu \mathrm{m}$ wide lines running along the probe shaft (figure 1(A)). Initial electrode impedances at $1 \mathrm{kHz}$ ranged $4.35 \pm 1.27 \mathrm{M} \Omega$, which could be lowered to $0.91 \pm 0.913 \mathrm{M} \Omega$ by additional gold electroplating.

The bonding pads on the probe base are connected to a highly flexible polyimide (PI) ribbon cable using a flip-chip bonding process [15]. The $10 \mu \mathrm{m}$ thin PI cable is further connected to a flexible printed circuit board (flex PCB) that fits to a small zero-insertion-force (ZIF) connector (Molex Inc., Lisle, Illinois, USA) (figure 1(A)). Sixteen ZIF connectors are integrated on a custom PCB that is mounted on top of the micro-drive array.

Probe fabrication. The silicon probes are fabricated on dualside polished 4 inch silicon wafers with a thickness of $300 \mu \mathrm{m}$ using standardized fabrication techniques established in micro-electromechanical systems engineering [16]. First, a $1 \mu \mathrm{m}$ thick, stress-compensated multi-layer stack of silicon oxide $\left(\mathrm{SiO}_{x}\right)$ and silicon nitride $\left(\mathrm{Si}_{x} \mathrm{~N}_{y}\right)$ is deposited using plasma enhanced chemical vapour deposition (PECVD). This layer serves as an electrical insulator between the probe metallisation and the underlying bulk silicon substrate. The metallisation itself is composed of titanium $(30 \mathrm{~nm}, \mathrm{Ti})$, gold $(200 \mathrm{~nm}, \mathrm{Au})$, platinum $(100 \mathrm{~nm}, \mathrm{Pt})$ and $\mathrm{Ti}(30 \mathrm{~nm})$ sputter deposited and patterned using a lift-off process. A second $1.5 \mu \mathrm{m}$ thick PECVD $\mathrm{SiO}_{x}-\mathrm{Si}_{x} \mathrm{~N}_{y}$ passivation layer protects the metallisation against the cerebral fluids in the brain tissue. 
(a)

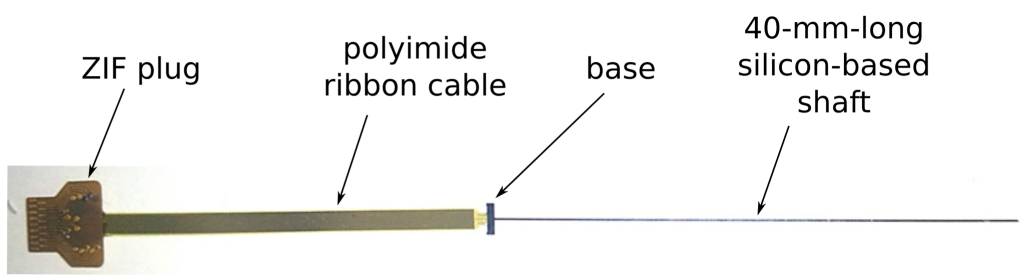

(b)



(c)

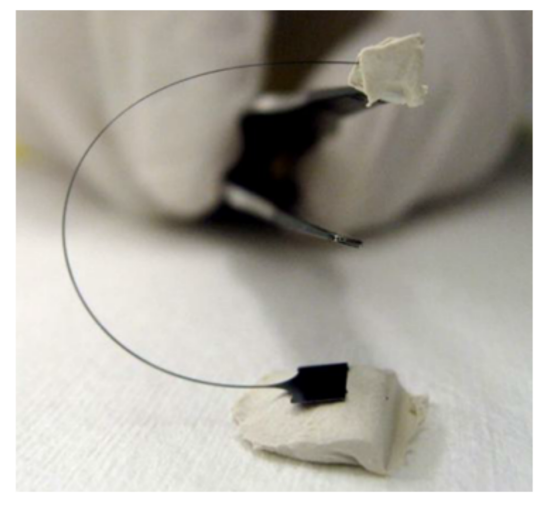

(d)
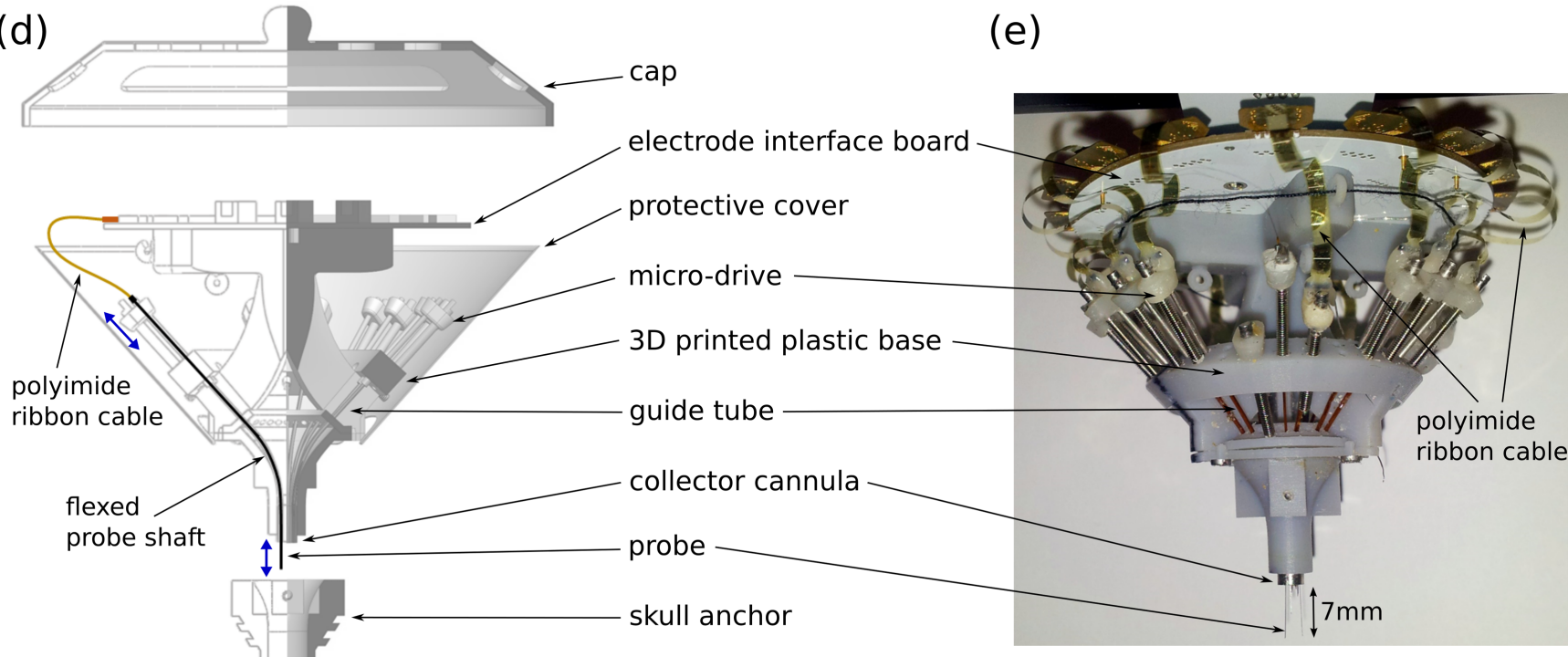

(e)

Figure 1. Integration of long, bendable silicon-based neural probes in a micro-drive array. (a) Photograph of fully assembled probe with silicon shaft, polyimide ribbon cable and zero-insertion force (ZIF) plug. (b) A close-up of the probe tip for the ' $D$ '-style and ' $L$ '-style electrode layouts. (c) Photograph demonstrating the flexibility of the silicon-based probe shank. (d) Cross-section (left: line drawing; right: shaded) of the 3D computer-aided design of the micro-drive array. The concept of a probe driven by an individually moveable micro-drive is shown on the left side. (e) Photograph of final array (without protective cover and cap) loaded with 16 probes. Two probes are fully extended from the bottom of the array. Note the polyimide ribbon cables that connect each probe to a ZIF connector on the electrode interface board.

Residual mechanical stress of the dielectric insulation and passivation layers has to be carefully compensated in order to minimise the bending of these ultra-thin probes [16], in particular in the case of the $40 \mathrm{~mm}$ long linear probes. Positive or negative wafer bows of more than $10 \mu \mathrm{m}$ measured after the deposition of the passivation layer using a wafer profilometer are compensated by additional thin layers of $\mathrm{Si}_{x} \mathrm{~N}_{y}$ or $\mathrm{SiO}_{x}$ of predefined thickness exploiting their tensile and compressive mechanical stress properties, respectively.

The PECVD passivation layer stack is patterned by reactive ion etching (RIE) to expose the electrode contacts along the probe shaft and the bonding pads on the probe base. This is followed by an ion beam etching step of the top $\mathrm{Ti}$ layer and gold sputter deposition of the electrode metallisation across the respective openings in the passivation layer. The electrode metallisation layer is again patterned using liftoff, resulting in electrodes with surface areas of either
$100 \mu \mathrm{m}^{2}$ or $225 \mu \mathrm{m}^{2}$. Following the definition of the electrodes, the passivation and insulation layers are patterned using RIE to achieve $40 \mu \mathrm{m}$ wide openings defining the probe geometry. Subsequently, $70 \mu \mathrm{m}$ deep trenches with vertical sidewalls are etched into the bulk silicon substrate using deep reactive ion etching where both PECVD layer stacks have been patterned. Finally, the wafers are thinned from the rear side using wafer grinding [16]. As soon as the trenches are reached during the grinding process step, the probes are released from the fabrication wafer. The grinding is combined with a dry etching process that removes the compressively stressed layer of the ground silicon surface [16] and produces straight $40 \mathrm{~mm}$ long probes. Further, as the surface is smoothened by the dry etching step, the mechanical yield of the probes under bending loads is improved. Final probes are assembled with the highly flexible PI ribbon cable using a flip-chip bonding process [15]. 


\section{Micro-drive array}

Micro-drive arrays are based on previous designs used for wire electrodes [10] and modified to fit up to 16 bendable probes and additional reference electrodes (figures 1(D) and (E)). Parts were designed in Solidworks CAD software (Dassault Systèmes SOLIDWORKS Corporation, Waltham MA, USA) and 3D printed in Accura Xtreme resin using a stereolithography process (Materialise, Leuven, Belgium).

The silicon shank of every probe (except for the bottom $7 \mathrm{~mm}$ ) was protected by a polyimide support tube (inner diameter $203 \mu \mathrm{m}$, outer diameter $259 \mu \mathrm{m}$ ) that was glued in place at the top of the shank close to the probe base. The support tube facilitates insertion into the micro-drive array and reduces the probability of accidental breaking. Each probe was mounted in a micro-drive using acrylate glue and light curable dental cement, and guided to the bottom of the array inside a polyimide tube (inner diameter $343 \mu \mathrm{m}$, outer diameter $497.5 \mu \mathrm{m}$ ). The probes were densely packed in the array and covered an area of approximately $2.6 \mathrm{~mm}$ in diameter.

Paths of the probes in the micro-drive array were carefully designed with a smooth curvature in a single plane to avoid sharp bends and twists that would increase the probability of probe malfunction. The length of the path was set such that probes extended $7 \mathrm{~mm}$ beyond the bottom of the array when micro-drives were in fully lowered position.

To facilitate recovery and reuse of an implanted microdrive array loaded with probes, the bottom part of the array was secured with screws to a single-use 3D-printed skull anchor (figure 1(D)). During implantation, only the skull anchor is embedded in dental cement and removal of the screws will release the micro-drive array at the end of an experiment.

The PI cables were maintained under the electronic interface board (EIB) with a string. The EIB contained additional connections for ground and reference electrodes. The complete array was protected by an aluminium-shielded and electrically grounded cover. Prior to the implantation, recording sites were gold plated (non-cyanide gold plating solution; Neuralynx, Bozeman, MT) using up to five $1 \mathrm{~s}$ long current pulses $(-0.1 \mu \mathrm{A})$ targeting an electrode impedance of $500 \mathrm{k} \Omega$ (NanoZ; White Matter LLC, Berkeley, CA). The guide tubes containing the probes were back-filled with mineral oil to prevent wicking up of biological fluids.

In comparison to wire electrodes, silicon probes are more brittle and prone to breaking when not handled appropriately. We found that with a proper technique as applied in this study, 16 probes can be assembled into a micro-drive array with very few fracture accidents within the same time needed to load a similar number of tetrodes.

\section{Surgery}

Two male Long-Evans rats were anaesthetized in an induction chamber with $5 \%$ isoflurane. Next, rats were mounted in a stereotaxic frame. During the entire procedure the anaesthesia level was maintained by $1 \%-2 \%$ isoflurane administered through a respiratory mask. Body temperature, heart rate and respiratory rate were continuously monitored (Kent Scientific Corporation, Torrington, CT). After sterilisation of the scalp with $10 \%$ iso-betadine and $70 \%$ isopropyl alcohol, a longitudinal incision exposed the underlying skull. Next, six anchoring bone screws were inserted into the skull, one of which served as the electrical ground. To implant the microdrive array, a $3.4 \mathrm{~mm}$ hole was drilled above the right hemisphere. In one rat, the implant was centred above hippocampal area CA1 at $3.6 \mathrm{~mm}$ posterior to Bregma and 2.4 from the midline. In a second rat, the implant was centred above area CA1 and subiculum at $4.4 \mathrm{~mm}$ posterior to Bregma and $2.3 \mathrm{~mm}$ from the midline. After removal of the dura, the micro-drive array (with all probes fully retracted) was positioned close to the cortical surface and any remaining exposed brain was protected with sterilised medical-grade silicone grease. Finally, the implant was secured in place using light curable dental cement. For 3 days following the surgery rats received subcutaneous injection of $0.5 \mathrm{ml}$ metacam $\left(5 \mathrm{mg} \mathrm{ml}^{-1}\right)$. All experiments were carried out in accordance with protocols approved by KU Leuven animal ethics committee in accordance with the European Council Directive, 2010/63/EU.

\section{Electrophysiology}

Wide band signals from up to 128 channels were sampled simultaneously and digitised at $32 \mathrm{kHz}$ per channel using a DigiLynx acquisition system (Neuralynx, Bozeman, MT). An adapter board exposed the 256 electrodes of all 16 probes through 8 Omnetics connectors compatible with DigiLynx headstages. Recordings from more than 128 channels (i.e. more than 8 probes) were performed sequentially in two adjacent sessions. After a period of post-surgical recovery, probes were slowly lowered into the brain. Signals were recorded either while rats were resting in a $60 \times 60 \mathrm{~cm}^{2}$ box, or while running back and forth on a linear track for food reward. Head position and direction were tracked at $25 \mathrm{~Hz}$ through a set of two light-emitting diodes mounted on the headstages and an overhead video camera.

\section{Data analysis}

Spike sorting. The classification of extracellular spike waveforms on each probe was performed using KlustaSuite, an open-source spike sorting software for multi-electrode recordings [17, 18]. Putative extracellular spikes were detected as threshold crossings in the high-pass filtered signals (500 Hz cut-off) in groups of neighbouring recording sites. A strong threshold of seven times the standard deviation (SD) of the filtered signal was used, corresponding to $73.0-100.5 \mu \mathrm{V}$ in different recording sessions. Signal samples crossing a weak threshold of $3.5 \mathrm{SD}$ on neighbouring electrodes within the same time period were considered to be originating from one spike. For each detected spike, a $2 \mathrm{~ms}$ long signal was captured around the centre of mass of the supra-threshold samples and 
summarised by the first three principal component features on each participating recording site.

In an initial automated step, spike sorting was performed using a masked expectation-maximisation algorithm [18]. A second manual stage was required to refine and correct the results of the automated step. This manual stage consisted of the following steps:

(1) Discard artefact clusters-Clusters with waveforms that were either highly similar on all recording sites or restricted to a single recording site, were discarded. Clusters with aberrant waveforms (i.e. either having many peaks or a single broad peak in the $2 \mathrm{~ms}$ time window) were discarded.

(2) Identify and split multi-unit clusters-Clusters were identified as multi-units based on the high number of inter-spike intervals smaller than $2 \mathrm{~ms}$ and/or on the presence of multiple visually distinct waveforms. A subset of multi-unit clusters could be manually split into clearly separated single units.

(3) Merge similar clusters-Two clusters were combined into one if they had very similar waveform shape and spatial distribution along the recording sites. Such clusters were initially split most likely because of small waveforms changes over short (e.g. bursts) or long time periods (e.g. behavioural state changes or electrode drift).

(4) Discard low rate and unstable units-Single units with mean firing rates $<0.05 \mathrm{~Hz}$ or units that appeared or disappeared during a session were excluded from further analysis.

Spatial tuning curves. In six recording sessions, rats ran back and forth along a $325 \mathrm{~cm}$ long L-shaped track for food reward. Linearised positions along the track were obtained by projecting the rat's location in the environment onto a handfitted spline model of the track. Velocity was computed from the linearised position and smoothed with a Gaussian kernel $(\mathrm{SD}=1 \mathrm{~s})$. For each isolated unit, a spatial tuning curve was constructed by computing the mean firing rate in $5 \mathrm{~cm}$ bins in both running directions and smoothing over position (Gaussian kernel, $\mathrm{SD}=10 \mathrm{~cm}$ ). Only times during which the rat ran at a speed of at least $10 \mathrm{~cm} \mathrm{~s}^{-1}$ were included.

\section{Histology}

After the experiment, rats were deeply anaesthetised with $3 \mathrm{ml}$ Nembutal $(60 \%)$ and electrolytic lesions were made using biphasic constant current pulses ( $30 \mu \mathrm{A}$ for $30 \mathrm{~s}$ ) on a selected number of probes and recording sites. To facilitate reconstruction of the location of all probes in the array, the lesions were made through five of the silicon probes located at the cardinal points and the centre of the collector cannula. A different number of recording sites were used for each probe in order to estimate the recording location of the probes. The rats were perfused through the heart with a phosphate buffered saline solution followed by a $4 \%$ paraformaldehyde solution. Brains were removed and sectioned in a cryotome (CM3050S, Leica) at $40 \mu \mathrm{m}$ thickness in the coronal plane. Brain sections were subjected to Nissl staining and inspected under the microscope.

\section{Results}

A maximum number of 16 bendable silicon-based neural probes could be integrated into a custom designed microdrive array. The whole device is $45 \mathrm{~mm}$ high with a maximum diameter of $72 \mathrm{~mm}$ and a weight of approximately $28 \mathrm{~g}$. Properly handled, the array behaves mechanically as similar devices loaded with wire electrodes. The flexible polyimide ribbon cables connected to the probes revealed to be light sensitive, such that strong changes in light intensity introduced large transients in the recorded signals. However, in the dimmed light conditions under which our experiments were performed, interference was minimal. To demonstrate the potential of the probe array to perform simultaneous highdensity recordings in distributed brain regions at various depths, probes were targeted to multiple cortical and/or hippocampal sites in two implanted rats.

In a first experiment (RAT 1), the array carried five functional probes, three of which were lowered to the cellular layer of hippocampal area CA1 while two probes were positioned in the overlying cortex. The tip of one of the probes ended up below the cell layer as the tissue recovered from the compressive action of pushing the probe down, and this probe was excluded from further analysis. The position of the other four probes was stable for the remainder of the experiment and only minimal readjustment (at most $60 \mu \mathrm{m} /$ day) was needed.

In the second experiment (RAT 2), an array of 16 probes was implanted and all probes were lowered to the cell layer of hippocampal areas CA1 or subiculum over multiple days. One probe broke while it was moved down into the brain. For the remaining 15 probes a mechanical interaction was observed such that when the depth of a single probe was adjusted, the signals of neighbouring probes were affected as well. The resulting instability of probe location across days required daily readjustment of subsets of probes and optimal positioning in the cell layer could not be obtained for all probes simultaneously. Probe tracks and electrolytic lesions made on selected electrodes for a subset of probes were readily visible in coronal brain sections (figure 2(A)). A reconstruction of the position of the probes in the brain showed that the array covered hippocampal area CA1 and subiculum (figure 2(B)).

Figure 3 shows an example of local field potentials recorded from a single ' $\mathrm{D}$ '-style probe positioned in the cellular layer and surrounding layers of area CA1. The rat was at rest, as also indicated by the presence of characteristic sharpwave ripple complexes [19] and associated bursts of spiking activity. 
(a)
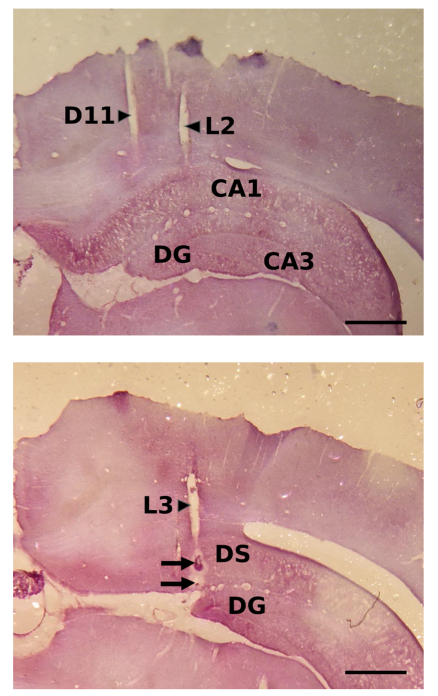

(b)



Figure 2. Histology and reconstruction of recording locations. (a) Two Nissl-stained coronal sections of RAT 2. Tracks of the shanks (arrowheads) and electrolytic lesions (arrows) were used to estimate the location of each probe. DG: dentate gyrus, DS: dorsal subiculum; scale bar: $1 \mathrm{~mm}$. (b) Reconstruction of the anterior-posterior and medial-lateral position of the probe array in RAT 2 overlaid on a top-view of 3D reconstructions of CA1 and subiculum (Atlas3D, Neural System and Graphic Computing Laboratory). Note, probe D10 was located close to the CA1-subiculum border, but since electrodes were facing posteriorly, D10 was assigned to subiculum. Grid scale: $1 \mathrm{~mm} \times 1 \mathrm{~mm}$.

(a)

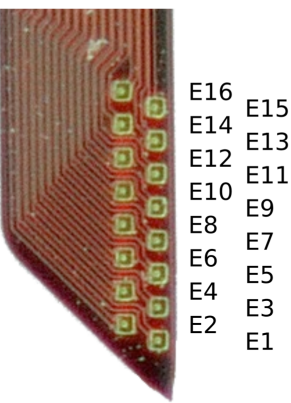

(b)

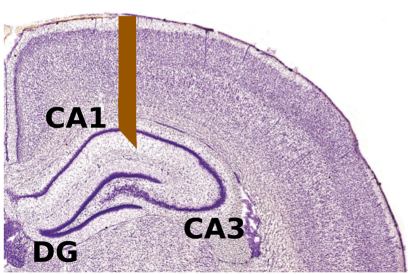

(c)

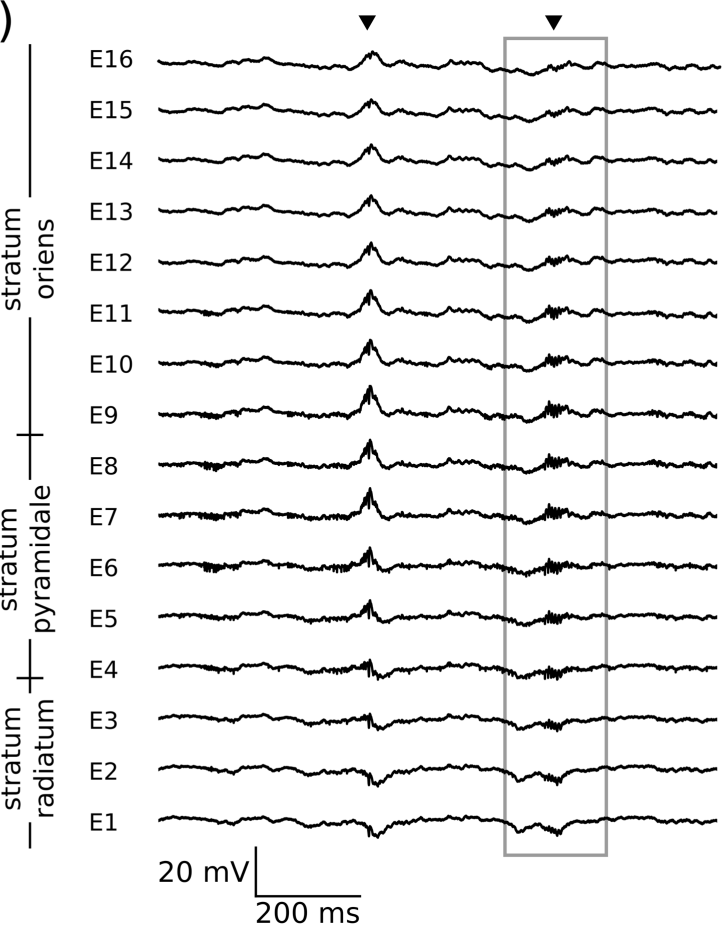

(d)

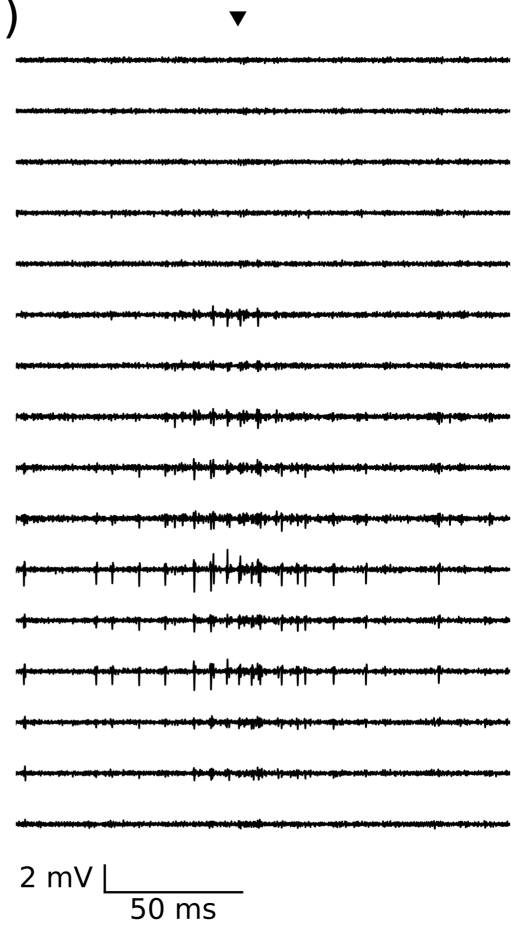

Figure 3. Local field potentials and unit activity. (a) Spatial distribution of electrodes on a 'D'-style probe and the correspondence to electrode sites (E1-E16). (b) Estimated location of a single probe in hippocampal area CA1 based on implantation coordinates and the distribution of local field potentials during sharp-wave ripple events. (c) Example of local field potentials recorded with a 'D'-style probe in and around the CA1 cell layer in RAT 1 (location as shown in (b)). Arrowheads indicate sharp-wave ripple events. Box indicates detail shown in (d). (d) Detail of local field potential traces around sharp-wave ripple event shown in (c). Signals were high-pass filtered with $500 \mathrm{~Hz}$ cut-off. Note the burst of spike activity co-occurring with the sharp-wave ripple event.

Well-isolated units that were stable for the duration of the recording session $\left(20-40 \mathrm{~min}\right.$ of rest in a $60 \times 60 \mathrm{~cm}^{2}$ box), were identified in both experiments and in all three sampled brain areas (figure $4(\mathrm{~A})$ ). The number of isolated units varied strongly across probes and days. In RAT 1, the number of units per probe ranged from 8 to 31 in hippocampus $\quad$ (mean $=15.7$ ) and 4 to 14 in neocortex (mean $=6.8$ ) during the first 9 days after placement of the 
(a)

unit \#



(b)
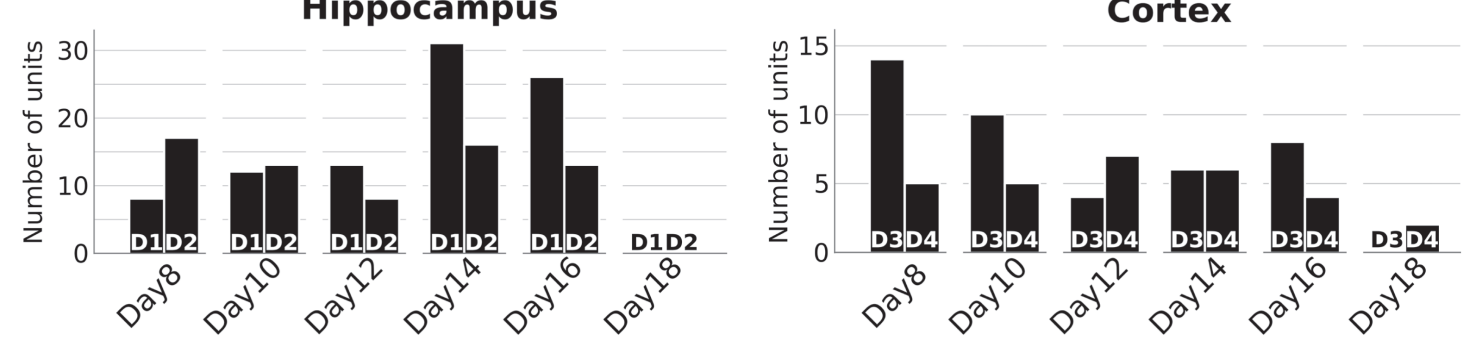

(c)

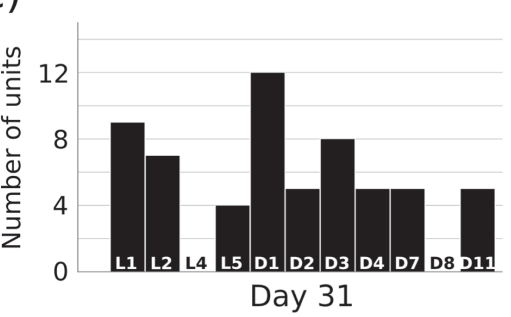

Hippocampus

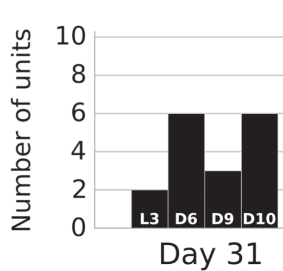

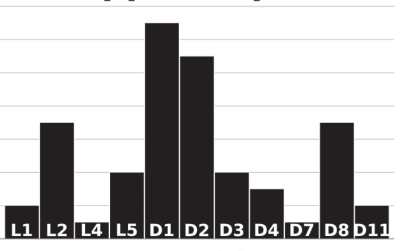

Day 33

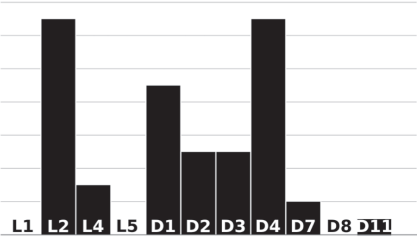

Day 34

\section{Subiculum}

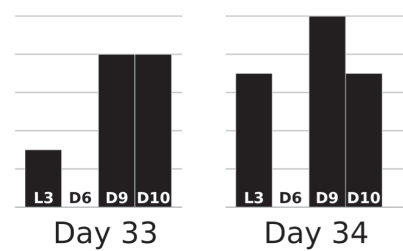

Figure 4. Counts of well-isolated single units across probes and days. (a) Spike waveforms for all well-isolated single units recorded from a single 'D'-style probe in CA1. Averaged waveforms (black) are overlaid on a random sample of single waveforms (grey density). Data obtained from probe D2 in RAT 1 on day 14. (b) Evolution of the number of well-isolated single units for two probes (D1 and D2) in hippocampus area CA1 (left) and two probes (D3 and D4) in neocortex (right) for RAT 1. Day 0 corresponds to the day the drive was implanted. (c) Number of well-isolated single units across 3 days for each of the 15 probes (L1-L5, D1-D4 and D5-D11) targeted at hippocampal area CA1 (top) and subiculum (bottom) in RAT 2. Day 0 corresponds to the day the drive was implanted. 
(a)

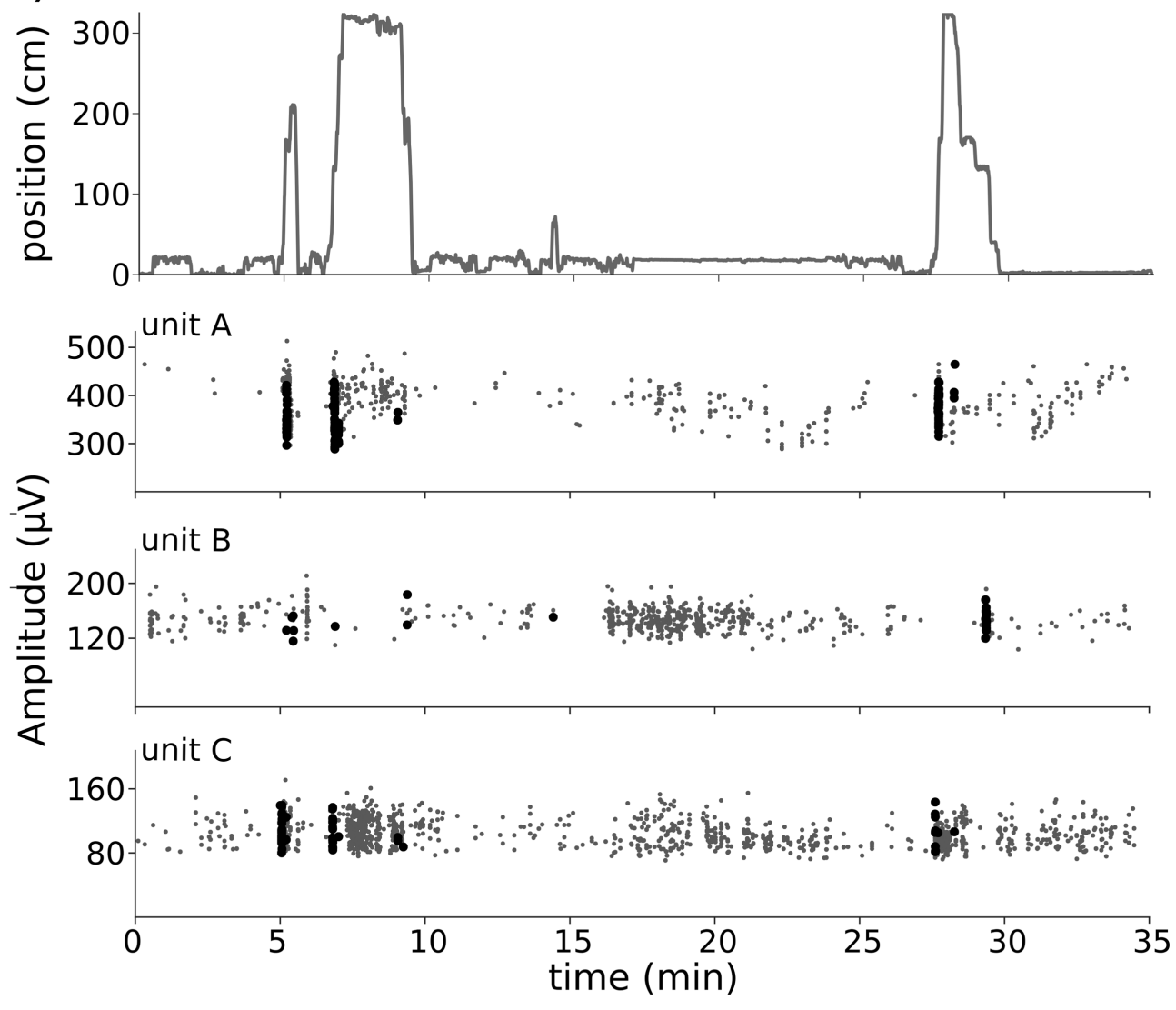

(b)

(c)
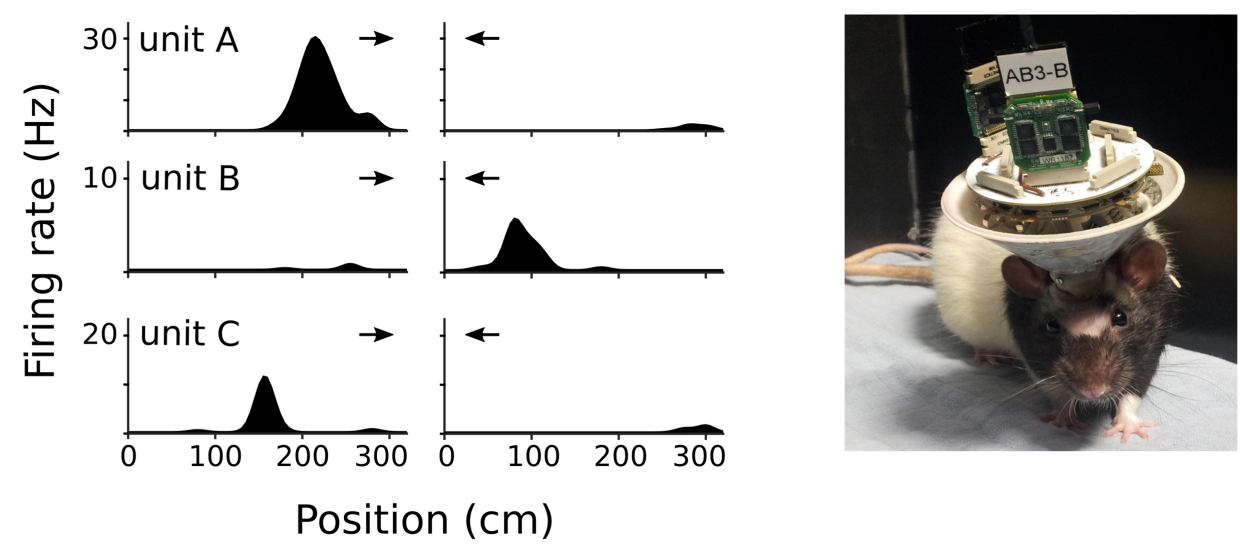

Figure 5. Stable recording of hippocampal place cells during active exploration. (a) Rat position on the track and variation over time of maximum spike amplitude for all spikes (grey) and spikes emitted during run epochs (black). Note that spike amplitudes were stable across the entire recording session. (b) Spatial and directional tuning of three selected units recorded from CA1 in RAT 2 while running along a $325 \mathrm{~cm}$ long track. Only spikes emitted during run epochs (speed $>10 \mathrm{~cm} \mathrm{~s}^{-1}$ ) were included. Arrows indicate running direction. (c) Photograph of rat with implanted micro-drive array carrying five bendable probes that are connected to the DigiLynx acquisition system through an adapter board.

probes in the targeted brain area (figure 4(B)). After 9 days there was a sudden strong drop in the number of units across all four probes.

In RAT 2, the number of isolated units ranged from 0 to 13 in CA1 (mean $=4.9)$ and 2 to 10 in subiculum $($ mean $=6)$ in 3 selected days about one month after the surgical implantation of the micro-drive array. Despite variations within individual probes, the total number of identified units recorded from all 15 probes was similar across days (74-78 units/ day; figure 4(C)). Stable recordings of isolated units were also obtained in RAT 1 while it actively explored a $325 \mathrm{~cm}$ long elevated track. Units in CA1 displayed spatial and directional tuning of their activity (figure 5), characteristic of place cells [20]. 


\section{Discussion}

\section{Large scale recordings with silicon probe array}

Chronic recordings in behaving rat. We describe a new recording system that integrates up to sixteen independently movable silicon probes into an implantable micro-drive array for chronic monitoring of neural activity in behaving animals. Each probe has 16 electrode contacts for a total of up to 256 recording sites per array. In our tests we recorded from a maximum of 128 electrodes at a time, and from all 256 electrodes sequentially in two groups, in freely behaving rats. Nevertheless, recent technical advances enable simultaneous recordings from up to 1024 electrodes [21-23], making highdensity recordings with more than 16 probes or more than 16 electrodes per probe feasible. The separate 3D-printed skull part that is a disposable interface between the micro drivearray and the dental cement is a novel feature in the design that facilitates re-use of the probe array and decreases turnaround time, when combined with proper probe cleaning procedures and optional reconditioning of the electrode sites.

Precise and flexible targeting of multiple brain regions. The main advantage of micro-drive arrays is that they offer the possibility to precisely target multiple probes in a tight bundle to cell populations at specific depths. This advantage has been exploited successfully with tetrodes in revealing population coding properties of hippocampal pyramidal cells that are arranged in a thin curved sheet [9, 24, 25]. Splitting the available electrodes across multiple sites, however, reduces the number of electrodes and hence the cell yield per brain region. This trade-off can be mitigated by the integration of silicon-based probes with a dense grid of electrodes into micro-drive arrays as demonstrated here. We performed distributed recordings with probes aimed at hippocampal and cortical sites located at different depths in one experiment and with probes spread across the cell layers of hippocampal areas CA1 and subiculum in a second experiment. The custom micro-drive array presented here was designed with a single circular bundle of probes that can reach brain structures up to $7 \mathrm{~mm}$ deep.

Further customization of the 3D-printed parts of the design with for example more than one bundle of electrodes supports distributed recording from multiple spatially distributed as well as deeper brain regions $[10,26]$. According to the needs of the experiment, a single array may also combine a variety of probe designs that incorporate high density recording electrodes, stimulation electrodes, drug delivery systems [27, 28] or light sources for optogenetic manipulations [29].

\section{Signal stability and longevity}

Chronic experiments with the array of bendable probes demonstrated that large numbers of cells can be recorded on individual probes $(>20$ in hippocampus CA1 and $\geqslant 10$ in neocortex and subiculum), even using a conservative spike sorting approach. Nevertheless, the variability across probes and across days is relatively high (in particular in the case of
16 implanted bendable probes in RAT 2), resulting in a low average number of cells as compared to arrays of wire electrodes [24] or fixed multi-shank probes [21]. There are a number of factors that affect to the short-term and long-term stability of the signals on the bendable probes in the array, including probe geometry, biocompatibility and method of insertion.

Probe geometry. The most likely explanation and limitation of the current probe design is the relatively large cross-section $\left(50 \times 150 \mu \mathrm{m}^{2}\right)$, which is larger than many available probes $[21,30]$. Given an inter-probe distance of $500 \mu \mathrm{m}$ in the bundle, the relative volume that is displaced locally is approximately $4 \%$. This volume displacement (and following slow relaxation of the brain tissue) is the likely cause of the observed signal instability over days, in particular in the second experiment in which the micro-drive array carried 16 probes in a $\sim 2.5 \mathrm{~mm}$ diameter bundle.

The large cross-section and resulting stiffness of the probes may also have contributed to the signal decline after 8-10 days, as compared to many weeks to months as reported previously for silicon-based or wire electrodes [31, 32]. Indeed, the larger the volume of an implant, the greater the compression of brain tissue and blood vessels resulting in haemorrhages, inflammatory and immune responses, and eventually neuronal loss $[6,33,34]$. Reducing the mechanical distortion of brain tissue by reducing the dimensions of the probe shank and improving the tip geometry will likely improve the quality of the recording. Indeed, reducing the width and thickness of the probe to below $100 \mu \mathrm{m}$ and $25 \mu \mathrm{m}$ respectively is attainable with the current state of technology. Even so, the long shank length of $4 \mathrm{~cm}$ likely imposes structural challenges during fabrication, which would necessitate further miniaturisation of the micro-drive array [35] to accommodate shorter bendable probes.

Biocompatibility. The chronic presence of a foreign body in the brain induces a long lasting reaction that is not the result of the initial mechanical trauma associated with the implantation. [36]. This response promotes macrophage and microglia proliferation and will typically lead to the formation of a dense glial scar around the shaft of the implant, therefore reducing the electrical coupling between the electrodes and the extracellular space, and neuronal death due to release of glial neurotoxic factors. Introduction of a dense array of neural probes likely exacerbates the immune response. Both reducing the physical dimensions of the probes and optimising the mode and speed of insertion may decrease the tissue response. In addition, surface treatment of the silicon probes with nanotubes or polymer coating to promote cell adhesion [37-39] or bioactive coating with antiinflammatory molecules or growth factors $[40,41]$ is also an approach that can be used in future devices to improve signal quality and longevity [42].

Method of insertion. The quality, stability and longevity of signals can also be increased by reducing tissue damage and 
dimpling through speed-controlled probe insertion [33, 43, 44]. Indeed, fast mechanical rather than manual insertion allows for longer lasting recordings [45]. As a future improvement, the hand-driven screws on the micro-drive array could be replaced by motorised micro-drives [46, 47] to provide such speed-controlled and more reliable positioning of the neural probes.

\section{Conclusion}

Current approaches to monitor neuronal activity either allow to flexibly record from multiple widely separated brain regions (micro-drive arrays) but with a limited sampling density or to provide denser sampling at the expense of a flexible placement in multiple brain regions (neural probes). The integration of state-of-the-art and next-generation probes into micro-drive array devices, as demonstrated here, offers an additional means of scaling for distributed recordings across multiple brain regions.

There are several opportunities for improving the bendable probe array. First, a reduction of the width and thickness of individual probes will reduce damage to brain tissue, which will likely translate into higher signal stability and cell yield. Second, the number of electrodes and their density in each probe can be increased to sample a larger volume and to increase the spatial sampling density which can improve single cell separation.

Most available silicon-based probe designs feature short shanks that are ideal for cortical recordings in small rodent brains, but not necessarily in larger (e.g. non-human primate) brains. To integrate silicon-based probes into a micro-drive array, the probe length had to be increased to match the length of the path inside the 3D-printed drive body. The same $4 \mathrm{~cm}$ long probes could also be used to record neural activity from deeper brain structures in large animal models.

\section{Acknowledgments}

We would like to thank Dun Mao, Daniela Schwindel, Keith Godfrey and Katarzyna Bzymek for their contribution and inputs. This project was partly supported by a grant from Alberta Innovates Health Solutions to BLM.

\section{Disclosures}

Arno Aarts and Tobias Holzhammer are co-founders of ATLAS Neuroengineering.

\section{References}

[1] Hebb D O 1949 The Organization of Behavior: A Neuropsychological Theory (New York: Wiley)
[2] Lewicki M S 1998 A review of methods for spike sorting: the detection and classification of neural action potentials Network 9 R53-78

[3] Gray C M, Maldonado P E, Wilson M and McNaughton B 1995 Tetrodes markedly improve the reliability and yield of multiple single-unit isolation from multi-unit recordings in cat striate cortex J. Neurosci. Methods 63 43-54

[4] Bragin A, Hetke J, Wilson C L, Anderson D J, Engel J and Buzsáki G 2000 Multiple site silicon-based probes for chronic recordings in freely moving rats: implantation, recording and histological verification J. Neurosci. Methods $9877-82$

[5] Lopez C M, Andrei A, Mitra S, Welkenhuysen M, Eberle W, Bartic C, Puers R, Yazicioglu R F and Gielen G G E 2014 An implantable 455-active-electrode 52-channel CMOS neural probe IEEE J. Solid-State Circuits 49 248-61

[6] Fekete Z 2015 Recent advances in silicon-based neural microelectrodes and microsystems: a review Sensors Actuators B 215 300-15

[7] Seidl K, Herwik S, Torfs T, Neves H P, Paul O and Ruther P 2011 CMOS-based high-density silicon microprobe arrays for electronic depth control in intracortical neural recording Microelectromech. Syst. J. 20 1439-48

[8] Blanche T J, Spacek M A, Hetke J F and Swindale N V 2005 Polytrodes: high-density silicon electrode arrays for largescale multiunit recording J. Neurophysiol. 93 2987-3000

[9] Wilson M A and McNaughton B L 1993 Dynamics of the hippocampal ensemble code for space Science 261 1055-8

[10] Kloosterman F, Davidson T J, Gomperts S N, Layton S P, Hale G, Nguyen D P and Wilson M A 2009 Micro-drive array for chronic in vivo recording: drive fabrication $J$. Vis. Exp. 26 e1094

[11] Miller E K and Wilson M A 2008 All my circuits: using multiple electrodes to understand functioning neural networks Neuron 60 483-8

[12] Perlin G E and Wise K D 2010 An ultra compact integrated front end for wireless neural recording microsystems J. Microelectromech. Syst. 19 1409-21

[13] Aarts A A A, Srivannavit O, Wise K D, Yoon E, Puers R, Van Hoof C and Neves H P 2011 Fabrication technique of a compressible biocompatible interconnect using a thin film transfer process J. Micromech. Microeng. 21074012

[14] Merriam M E, Dehmel S, Srivannavit O, Shore S E and Wise K D 2011 A 3-d 160-site microelectrode array for cochlear nucleus mapping IEEE Trans. Biomed. Eng. 58 397-403

[15] Kisban S, Kenntner J, Janssen P, Metzen R V, Herwik S, Bartsch U, Stieglitz T, Paul O and Ruther P 2009 A novel assembly method for silicon-based neural devices IFMBE Proc. 25/9 107-10

[16] Herwik S, Paul O and Ruther P 2011 Ultrathin silicon chips of arbitrary shape by etching before grinding J. Microelectromech. Syst. 20 791-3

[17] Rossant C et al 2016 Spike sorting for large, dense electrode arrays Nat. Neurosci. 19 634-41

[18] Kadir S N, Goodman D F M and Harris K D 2014 Highdimensional cluster analysis with the masked EM algorithm Neural Comput. 26 2379-94

[19] Buzsáki G 1986 Hippocampal sharp waves: their origin and significance Brain Res. 398 242-52

[20] O'Keefe J 1976 Place units in the hippocampus of the freely moving rat Exp. Neurol. 51 78-109

[21] Berényi A, Somogyvári Z, Nagy A J, Roux L, Long J D, Fujisawa S, Stark E, Leonardo A, Harris T D and Buzsáki G 2014 Large-scale, high-density (up to 512 channels) recording of local circuits in behaving animals J. Neurophysiol. 111 1132-49

[22] Shobe J L, Claar L D, Parhami S, Bakhurin K I and Masmanidis S C 2015 Brain activity mapping at multiple 
scales with silicon microprobes containing 1024 electrodes J. Neurophysiol. 114 2043-52

[23] Scholvin J, Kinney J P, Bernstein J G, Moore-Kochlacs C, Kopell N, Fonstad C G and Boyden E S 2016 Close-packed silicon microelectrodes for scalable spatially oversampled neural recording IEEE Trans. Biomed. Eng. 63 120-30

[24] Pfeiffer B E and Foster D J 2013 Hippocampal place-cell sequences depict future paths to remembered goals Nature 497 74-9

[25] Diba K and Buzsáki G 2007 Forward and reverse hippocampal place-cell sequences during ripples Nat. Neurosci. 10 1241-2

[26] Headley D B, DeLucca M V, Haufler D and Paré D 2015 Incorporating 3D-printing technology in the design of headcaps and electrode drives for recording neurons in multiple brain regions J. Neurophysiol. 113 2721-32

[27] Pongrácz A, Fekete Z, Márton G, Bérces Z, Ulbert I and Fürjes P 2013 Deep-brain silicon multielectrodes for simultaneous in vivo neural recording and drug delivery Sensors Actuators B. 189 97-105

[28] Lee H J, Son Y, Kim D, Kim Y K, Choi N, Yoon E-S and Cho I-J 2015 A new thin silicon microneedle with an embedded microchannel for deep brain drug infusion Sensors Actuators B 209 413-22

[29] Wu F, Stark E, Im M, Cho I-J, Yoon E-S, Buzsáki G, Wise K D and Yoon E 2013 An implantable neural probe with monolithically integrated dielectric waveguide and recording electrodes for optogenetics applications J. Neural Eng. 10056012

[30] Csicsvari J, Henze D A, Jamieson B, Harris K D, Sirota A, Barthó P, Wise K D and Buzsáki G 2003 Massively parallel recording of unit and local field potentials with silicon-based electrodes J. Neurophysiol. 90 1314-23

[31] Williams J C, Rennaker R L and Kipke D R 1999 Long-term neural recording characteristics of wire microelectrode arrays implanted in cerebral cortex Brain Res. Protocols $\mathbf{4}$ 303-13

[32] Kipke D R, Vetter R J, Williams J C and Hetke J F 2003 Silicon-substrate intracortical microelectrode arrays for long-term recording of neuronal spike activity in cerebral cortex IEEE Trans. Neural Syst. Rehabil. Eng. 11 151-5

[33] Sharp A A, Ortega A M, Restrepo D, Curran-Everett D and Gall K 2009 In vivo penetration mechanics and mechanical properties of mouse brain tissue at micrometer scales IEEE Trans. Biomed. Eng. 56 45-53

[34] Andrei A, Welkenhuysen M, Nuttin B and Eberle W 2012 A response surface model predicting the in vivo insertion behavior of micromachined neural implants J. Neural Eng. 9 016005

[35] Voigts J, Siegle J H, Pritchett D L and Moore C I 2013 The flexDrive: an ultra-light implant for optical control and highly parallel chronic recording of neuronal ensembles in freely moving mice Front. Syst. Neurosci. 78

[36] Polikov V S, Tresco P A and Reichert W M 2005 Response of brain tissue to chronically implanted neural electrodes J. Neurosci. Methods 148 1-18

[37] Cui X, Lee V A, Raphael Y, Wiler J A, Hetke J F, Anderson D J and Martin D C 2001 Surface modification of neural recording electrodes with conducting polymer/ biomolecule blends J. Biomed. Mater. Res. 56 261-72

[38] Fan Y, Cui F, Chen L, Zhai Y, Xu Q and Lee I-S 2002 Adhesion of neural cells on silicon wafer with nanotopographic surface Appl. Surf. Sci. 187 313-8

[39] Keefer E W, Botterman B R, Romero M I, Rossi A F and Gross G W 2008 Carbon nanotube coating improves neuronal recordings Nat. Nanotechnol. 3 434-9

[40] Zhong Y, Yu X, Gilbert R and Bellamkonda R V 2001 Stabilizing electrode-host interfaces: a tissue engineering approach J. Rehabil. Res. Dev. 38 627-32

[41] Grand L et al 2010 Short and long term biocompatibility of NeuroProbes silicon probes J. Neurosci. Methods 189 216-29

[42] Aregueta-Robles U A, Woolley A J, Poole-Warren L A, Lovell N H and Green R A 2014 Organic electrode coatings for next-generation neural interfaces Front. Neuroeng. 715

[43] Bjornsson C S, Oh S J, Al-Kofahi Y A, Lim Y J, Smith K L, Turner J N, De S, Roysam B, Shain W and Kim S J 2006 Effects of insertion conditions on tissue strain and vascular damage during neuroprosthetic device insertion $J$. Neural Eng. 3 196-207

[44] Hosseini N H, Hoffmann R, Kisban S, Stieglitz T, Paul O and Ruther P 2007 Comparative study on the insertion behavior of cerebral microprobes Proc. 29th Annu. Int. Conf. of the IEEE Engineering in Medicine and Biology Society, IEEE EMBS vol 2007 pp 4711-4

[45] Rennaker R L, Street S, Ruyle A M and Sloan A M 2005 A comparison of chronic multi-channel cortical implantation techniques: manual versus mechanical insertion J. Neurosci. Methods 142 169-76

[46] Otchy T M and Ölveczky B P 2012 Design and assembly of an ultra-light motorized microdrive for chronic neural recordings in small animals J. Vis. Exp. 69 e4314

[47] Yamamoto J and Wilson M A 2008 Large-scale chronically implantable precision motorized microdrive array for freely behaving animals J. Neurophysiol. 100 2430-40 